\title{
GLANDULAR CYSTITIS - A RARE BENIGN CONDITION PRESENTING AS A PSEUDO-TUMOR OF THE BLADDER
}

\author{
Anuar Ibrahim Mitre, Carlos Alberto Paes Silveira, Kátia Ramos Moreira Leite, Affonso Celso Piovesan
}

\section{INTRODUCTION}

There are conditions that simulate bladder tumors from the standpoint of clinical manifestation, imaging, and endoscopic findings. One of these, glandular cystitis due to inflammation of the lamina propria and proliferation of the epithelium by invagination, can cause macroscopic alterations suggestive of a neoplasm. There is no therapeutic recommendation because of the small number of cases that have been previously published and because its etiopathogenesis is unknown. We report how the diagnosis of glandular cystitis was made as well as the treatment strategy.

\section{CASE PRESENTATION AND MANAGEMENT}

A 28-year-old white male had undergone ultrasonography, which revealed an irregularity in the vesical floor (Figure 1). He complained of obstructive and irritative miction symptoms without hematuria. The urine test was normal. On digital rectal examination, a solid mass above the prostate was detected between the rectum and the bladder. Computed tomography confirmed the findings of ultrasonography and in addition revealed bilateral ureteral ectasis (Figure 2). The patient underwent endoscopic resection

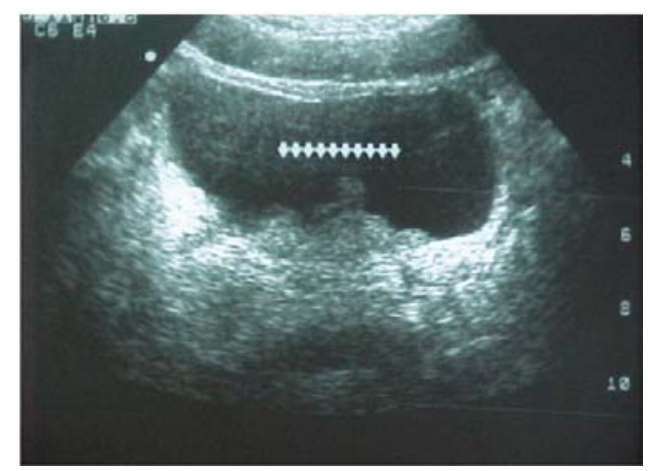

Figure 1 - Ultrasonography showing irregularities at the base of the bladder suggesting a bladder tumor

Department of Urology, São Paulo University Medical School - São Paulo/ SP, Brazil.

Email: anuar@mitre.com.br of a sessile tumor in the prostatic urethra, bladder neck, trigone, and portions of the lateral walls of the bladder. The histological examination revealed cystic glandular cystitis with extensive areas presenting an enteric pattern, without signs of malignancy (Figure 3). Ultrasonography of the urinary tract carried out 2 months postoperatively showed vesical thickening and irregularity in the area of the bladder floor, but without bilateral ureteral dilation. In another endoscopic procedure, we again resected vegetating tissue found in the area of the trigone, bladder neck, and prostatic urethra. The histologic examination confirmed the diagnosis of glandular cystitis.

Due to recurrence of the pseudo-tumoral tissue, and in the absence of a pre-established therapeutic course, we decided to treat the condition using intravesical BCG (bacillus Calmette-Guerin) with a weekly application for a total
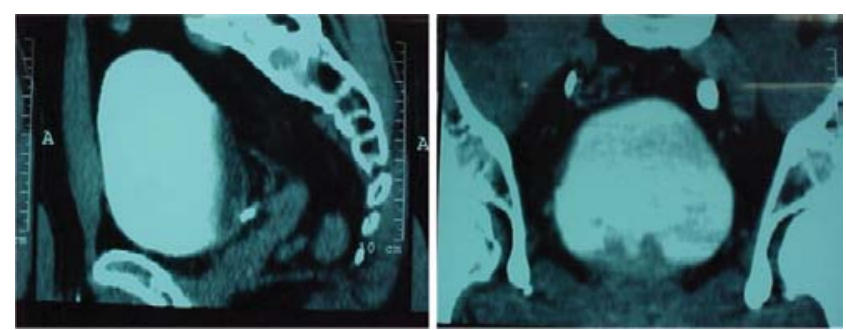

Figure 2 - Computed tomography indicating a mass between the bladder and the rectum, in a sagittal section, and at the base of the bladder in a frontal section
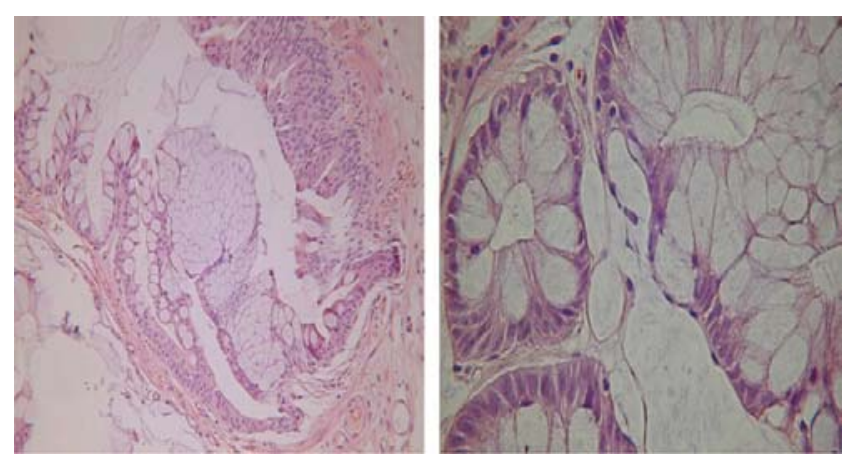

Figure 3 - (A) Microphotograph showing the transitional area between the normal urothelium and the metaplastic glandular epithelium with an enteric pattern (B) High cylindric cells with polarized nuclei inferiorly, large cytoplasm, clear, and full of mucus. 
of 8 doses, beginning 2 months after the last endoscopic resection.

The ultrasonographic control after a year indicated slight ureteropyelocaliceal dilatation, which was more pronounced at the right distal ureter, and a thickening of the bladder wall.

A new resection of the irregularity of the bladder neck mucosa and vesical trigone revealed glandular cystitis. The patient underwent another series of BCG treatments.

Two years later the patient presented no voiding complaints, urinary flow was better than before, and ejaculations were normal.

Cultures of urine and urinary cytology were normal. The cystoscopy showed irregular urothelium at the bladder neck and trigone, and the biopsy of these areas did not reveal the previous abnormal findings.

\section{DISCUSSION}

The metaplastic alterations of the vesical transitional epithelium in glandular cystitis are well documented in the literature, although the etiology is not yet completely clarified. ${ }^{1-3}$ It is believed that glandular cystitis is due to chronic inflammation of the lamina propria possibly resulting from the prolonged exposure to irritating agents.

Chronic inflammation of the lamina propria causes proliferation of the epithelium resulting in invagination, with formation of nests, crypts, and, in some cases, actual enteric glands. It can be preceded or associated with other prolifer ative non-neoplastic lesions such as Von Brunn nests, cystic cystitis, follicular cystitis, and polypoid cystitis.

Unlike the glandular cystitis, polypoid cystitis arises from the extrinsic proliferation of the epithelium above the lamina propria, with formation of small villous projections. It can remain as a benign process for years, or it can turn into an autonomous growth and produce a solid papillary invasive tumor. ${ }^{3}$

Investigating autopsy samples, Morse ${ }^{4}$ concluded that these epithelial alterations are more common in people over 20 years and that they would problably be related to chronic inflammations of the urinary tract, ${ }^{2,5}$ as can be observed in schistosomiasis. ${ }^{6}$

Another etiologic hypothesis is based on embryonal remnants that originate in the urogenital sinus leading to the formation of actual enteric glands, with or without mucosal contents in the bladder. Metaplasias of the vesical mucosa can also result from embryonal remains of skin (scamous metaplasia), or from a primitive kidney (nephrogenic adenomas). ${ }^{2}$

Some authors consider glandular cystitis to be a histologic curiosity without any clinical significance. ${ }^{1}$ However, a great majority have considered it to be a premalignant lesion with an early histologic correlation to less common types of vesical neoplasms..$^{2,5-8}$ The frequent association of these proliferative lesions with carcinomas in situ or with invasive neoplasms reinforces the hypothesis that glandular cystitis is a precancerous lesion. ${ }^{8}$

The association of glandular cystitis with pelvic lipomatosis has been described. ${ }^{9}$

Regarding the risk of glandular cystitis progressing to malignancy, there are studies that demonstrate a correlation between the reactivity of the antibody mAbDas 1 with columnar mucinous adenocarcinoma and glandular metaplasia. ${ }^{10}$ This marker might be useful for determining the risk of progression to malignancy of the intestinal metaplasia.

In the incidentally diagnosed case that we are presently reporting, glandular cystitis presented as a pseudo-tumoral lesion. Because of the recurrence of lesions and of possible correlations with a malignant neoplasm, we chose to perform endoscopic resections and, following the procedure, to treat the condition using intravesical BCG until these histological alterations had disappeared.

\section{REFERENCES}

1. Catalona W. Urothelial tumors of the urinary tract. In: Walsh PC. Campbell's urology, 6th ed. vol 2. Philadelphia: Saunders; 1992. p. 1094158.

2. Erturk E, Erturk E, ScheinfeldJ, Davis RS. Metaplastic cystitis complicated with Von Brunn nests, cystitis cystica and intestinal type of glandular metaplasia. Urology. 1988;32:165-7.

3. Mostofi FK. Potentialities of bladder epithelium. J Urol. 1954;71:70514.

4. Morse H.D. The etiology and pathology of pyelitis cystica, ureteritis cystica and cystitis cystica. Am J Pathol. 1928;4:33.

5. Kittredge WE, Collett AJ, Morgan C. Adenocarcinoma of the bladder associated with cystitis glandularis: a case report. J Urol. 1964;91:145.
6. Salm, R. Neoplasia of the bladder and cystitis cystica. Br J Urol. 1967;39:67-72.

7. Blake J. Proliferative changes in bladder mucosa and carcinoma in situ. Br J Urol. 1967;39:181-5.

8. Shaw JL, Gislason G J and Imbriglia JE. Transition of cystitis glandularis to primary adenocarcinoma of the bladder. J Urol. 1958;79:815-22.

9. Resnick MI, and Kursh ED. Extrinsic obstruction of the ureter. In: Walsh PC, editor. Campbell's urology, 6th ed, vol 1 .Philadelphia: Saunders; 1992. p. $533-70$.

10. Murphy DP, Pantuck AJ, Amenta PS, Das KM, Cummings KB, Keeney GL, et al. Female urethral adenocarcinoma: immunohistochemical evidence of more than 1 tissue of origin. J Urol. 1999;161:1881-4. 